\title{
Strain-induced switching between noncollinear and collinear spin configuration in magnetic $\mathrm{Mn}_{5} \mathrm{Ge}_{3}$ films
}

Yufang Xie ${ }^{1,4, *}$, Ye Yuan ${ }^{2,9}$, Magdalena Birowska ${ }^{3}$, Chenhui Zhang ${ }^{2}$, Lei Cao ${ }^{1}$, Mao Wang ${ }^{1}$, Joerg Grenzer $^{1}$, Dominik Kriegner ${ }^{5,6}$, Petr Doležal ${ }^{7}$, Yu-Jia Zeng ${ }^{8}$, Xixiang Zhang ${ }^{2}$, Manfred Helm $^{1,4}$, Shengqiang Zhou ${ }^{1}$, Slawomir Prucnal ${ }^{1, \dagger}$

${ }^{1}$ Institute of Ion Beam Physics and Materials Research, Helmholtz-Zentrum DresdenRossendorf, Bautzner Landstrasse 400, D-01328 Dresden, Germany

${ }^{2}$ Physical Science and Engineering Division, King Abdullah University of Science and Technology, 23955-6900 Thuwal, Saudi Arabia

${ }^{3}$ Faculty of Physics, Institute of Theoretical Physics, University of Warsaw, Pasteura 5, PL02093 Warsaw, Poland

${ }^{4}$ Technische Universität Dresden, D-01062 Dresden, Germany

${ }^{5}$ Institut für Festkörper- und Materialphysik and Würzburg-Dresden Cluster of Excellence ct.qmat, Technische Universität Dresden, D-01062 Dresden, Germany

${ }^{6}$ Institute of Physics, Czech Academy of Sciences, Cukrovarnická 10, 16200 Praha 6, Czech Republic

${ }^{7}$ Charles University, Faculty of Mathematics and Physics, Department of Condensed Matter Physics, Ke Karlovu 5, 12116 Prague 2, Czech Republic

${ }^{8}$ College of Physics and Optoelectronic Engineering, Shenzhen University, Shenzhen 518060, People's Republic of China

${ }^{9}$ Songshan Lake Materials Laboratory, Dongguan, Guangdong 523808, People's Republic of China

*y.xie@hzdr.de

†s.prucnal@hzdr.de 
We report the temperature-dependent magnetic and structural properties of epitaxial $\mathrm{Mn}_{5} \mathrm{Ge}_{3}$ thin films grown on Ge substrates. Utilizing density-functional theory (DFT) calculations and various experimental methods, we reveal mechanisms governing the switching between collinear and noncollinear spin configuration in $\mathrm{Mn}_{5} \mathrm{Ge}_{3}$. The $\mathrm{Mn}$ atoms in $\mathrm{Mn}_{5} \mathrm{Ge}_{3}$ occupy two distinct Wyckoff positions with fourfold $\left(\mathrm{Mn}_{1}\right)$ and sixfold $\left(\mathrm{Mn}_{2}\right)$ multiplicity. The DFT calculations reveal that below a critical distance of approximately $3.002 \AA$ the coupling between $\mathrm{Mn}_{2}$ atoms is antiferromagnetic (AFM) while ferromagnetic (FM) above that critical distance. The FM coupling between $\mathrm{Mn}_{1}$ atoms is weakly affected by the strain. The observed noncollinear spin configuration is due to the coexistence of AFM and FM coupling at low temperatures. The findings give insight in developing strain controlled spintronic devices. 


\section{INTRODUCTION}

Spintronic offers lower power dissipation and processing capabilities much beyond the current Complementary Metal Oxide Semiconductor technology [1,2]. However, it is very challenging to find a ferromagnetic semiconductor material with high Curie temperature $\left(T_{C}\right)$ and compatible with $\mathrm{Si}$ technology. Ferromagnetic $\mathrm{Mn}_{5} \mathrm{Ge}_{3}$ films fabricated by epitaxial solid-state reaction on Ge substrates exhibit a $T_{C}$ of about $283 \mathrm{~K}$ [3], a considerable spin polarization [4,5], a sharp interface between the ferromagnetic layer and Ge substrates [6,7], all of which are essential to achieve efficient spin injection and manipulation within semiconductors prepared by already-existing silicon-based technologies. Furthermore, the $T_{C}$ of $\mathrm{Mn}_{5} \mathrm{Ge}_{3}$ was shifted much above room temperature by applying strain engineering ( $320 \mathrm{~K})$ [8], quantum confinement ( 400 K) [9] or co-doping with carbon ( 430 K) [10,11]. The fundamental properties of $\mathrm{Mn}_{5} \mathrm{Ge}_{3}$, especially the magnetic structure, have been investigated in detail [1214]. $\mathrm{Mn}_{5} \mathrm{Ge}_{3}$ possesses a hexagonal $D 8_{8}$-type crystal structure with space group $P 6_{3} / \mathrm{mcm}$ whose unit cell contains $6 \mathrm{Ge}$ and $10 \mathrm{Mn}$ atoms. The Mn atoms occupy two different sublattices where $\mathrm{Mn}_{1}$ is located at the Wyckoff $4 d$ site and $\mathrm{Mn}_{2}$ at the $6 g$ site with $\mathrm{x}=0.2397$ [14]. In the relaxed unit cell, the magnetic moment of $\mathrm{Mn}_{1}$ is $1.96 \mu_{B} / \mathrm{Mn}$ and that of $\mathrm{Mn}_{2}$ is $3.23 \mu_{B} / \mathrm{Mn}$ [14]. The magnetic moment direction of $\mathrm{Mn}_{1}$ and $\mathrm{Mn}_{2}$ atoms has been demonstrated to be parallel to the $c$ axis of the hexagonal structure from $77 \mathrm{~K}$ to $T_{C}$. The distinguished magnetic coupling between two different sublattices leads to an anisotropic exchange and complex magnetic ordering in different temperature regimes.

$A b$ initio pseudopotential calculations have demonstrated that $\mathrm{Mn}_{5} \mathrm{Ge}_{3}$ has two competing phases with collinear and noncollinear spin configurations [15]. The interaction $\left(J_{1}\right)$ between the nearest neighbors $\mathrm{Mn}_{1}-\mathrm{Mn}_{1}$ is ferromagnetic and weakly depends on the distance $\left(d_{1}\right)$ between the $\mathrm{Mn}_{1}$ atoms, and it is much stronger than the interaction between the $\mathrm{Mn}_{1}-\mathrm{Mn}_{2}$ and $\mathrm{Mn}_{2}-\mathrm{Mn}_{2}$ atoms [16]. In contrast, the interaction $\left(J_{3}\right)$ between the nearest neighbors $\mathrm{Mn}_{2}-\mathrm{Mn}_{2}$ strongly depends on the related atomic distance $\left(d_{3}\right)$. The neighboring $\mathrm{Mn}_{2}$ atoms are ferromagnetically (FM) coupled in the fully relaxed unit cell where $d_{3}=3.017 \AA$ [based on density-functional theory (DFT) calculation]. By applying compressive strain, $d_{3}$ decreases and the corresponding FM coupling is suppressed then transformed into antiferromagnetic (AFM) coupling. In theoretical calculations presented by Slipukhina et al. [16], when $d_{3}=2.974 \AA$, the neighbor $\mathrm{Mn}_{2}$ atoms are AFM coupled. The competing interactions between the AFM coupling between $\mathrm{Mn}_{2}$ and the $\mathrm{FM}$ coupling between $\mathrm{Mn}_{1}$ atoms stabilize noncollinear spin 
configurations $[17,18]$. The transition temperature from collinear to noncollinear magnetism in $\mathrm{Mn}_{5} \mathrm{Ge}_{3}$ is $70 \pm 5 \mathrm{~K}[19,20]$ which is usually explained by similar lattice distortions observed in $\mathrm{Mn}_{5} \mathrm{Si}_{3}$ [21-23]. Yet there is no convincing explanation, why the noncollinear spin configuration appears in nanostructured $\mathrm{Mn}_{5} \mathrm{Ge}_{3}$ but not in bulk materials $[16,19,20]$.

To gain insight into the complex physical properties of ferromagnetic $\mathrm{Mn}_{5} \mathrm{Ge}_{3}$ thin films, we have performed systematically experimental investigation using temperature and angulardependent magnetoresistance, temperature-dependent x-ray diffraction (TDXRD) as well as theoretical calculations using DFT [24-26]. According to our theoretical calculations the exchange constant $J_{3}$ becomes negative and the nearest-neighboring $\mathrm{Mn}_{2}-\mathrm{Mn}_{2}$ atoms are AFM coupled for a $d_{3}^{D F T}$-value on the order of $3.002 \AA$ or smaller. The relative distance $d_{3}^{\text {exp }}$ between two neighbor $\mathrm{Mn}_{2}$ atoms, extracted experimentally from TDXRD, shrinks from 3.012 $\pm 0.002 \AA$ at room temperature down to $2.999 \pm 0.002 \AA$ at $5 \mathrm{~K}$. The critical distance for the neighboring $\mathrm{Mn}_{2}$ atoms ( $d_{3}$ smaller than $3.002 \AA$ ) at which magnetic coupling changes from FM to AFM is achieved at the temperature of about $150 \pm 10 \mathrm{~K}$. This means that at low temperatures the AFM coupling of $\mathrm{Mn}_{2}$ atoms coexists with FM coupling of $\mathrm{Mn}_{1}$ atoms. At higher temperatures, all coupling interaction between $\mathrm{Mn}$ atoms is ferromagnetic. The temperature dependent anisotropic magnetoresistance (AMR) reveals a well-visible transition from twofold to multifold symmetry with increasing temperature. This work provides experimental and theoretical evidence that both the switching between noncollinear and collinear spin configurations and the intriguing behavior of AMR are caused by the change of the magnetic coupling between $\mathrm{Mn}_{2}$ atoms occupying $6 g$ positions in the $\mathrm{Mn}_{5} \mathrm{Ge}_{3}$ sublattice.

\section{RESULTS AND DISCUSSION}

\section{A. Magnetotransport results}

In this work, (100)-orientated $\mathrm{Mn}_{5} \mathrm{Ge}_{3}$ epitaxial films were fabricated on (001) Ge substrates by ms-range solid-state reaction (see the inset in Fig. 1) [3]. Figure 1 shows the temperaturedependent resistivity $(\rho-T)$ and the related first derivative curve of the $\mathrm{Mn}_{5} \mathrm{Ge}_{3}$ thin film. In the $d \rho / d T$ curve, a clear transition from positive to negative near $283 \mathrm{~K}$ is observed, which mainly ascribed to the transition from ferromagnetic to paramagnetic state $[3,6,7,27]$. The observed $T_{C}$ corresponds well to the value obtained by superconducting quantum interference device-vibrating-sample magnetometer measurements (see Fig. S1 in Supplemental Material [28]). Below $T_{C}(283 \mathrm{~K})$ the resistivity decreases with decreasing temperature, showing typical 
metallic behavior $[16,29,30]$. Below $T_{C}$, the carrier-magnon scattering must be taken into account as well since the electron-magnon scattering dominates the electrical transport properties in strong ferromagnetic films.

It is worth noting there is another small deformation (kind of cusp) in the $d \rho / d T$ curve at $T_{I} \sim$ $72 \mathrm{~K}$ beside the well-visible transition at $T_{C}$. Such a cusp is commonly observed in $\mathrm{Mn}_{5} \mathrm{Ge}_{3}$ thin films and nanostructures but has not been shown in bulk crystals $[19,20,31]$. The appearance of such a cusp in the $d \rho / d T$ curve is often related to the emergence of a new magnetic ordering. Zeng et al. reported that the cusp is due to the coexistence of two magnetic sublattices $\left(\mathrm{Mn}_{1}\right.$ and $\mathrm{Mn}_{2}$ ) with different magnetocrystalline anisotropies and exchange coupling constants [19]. The change of the magnetic coupling between $\mathrm{Mn}$ atoms was concluded by an analogy to the antiferromagnetic $\mathrm{Mn}_{5} \mathrm{Si}_{3}$ where noncollinear spin states exist at lower temperatures [21,23,32]. The nontrivial spin arrangements at lower temperatures were demonstrated to induce the topological Hall effect $[23,33]$. To date, no further interpretation has been proposed to understand the underlying mechanism why the noncollinear spin configuration exists in $\mathrm{Mn}_{5} \mathrm{Ge}_{3}$ films.

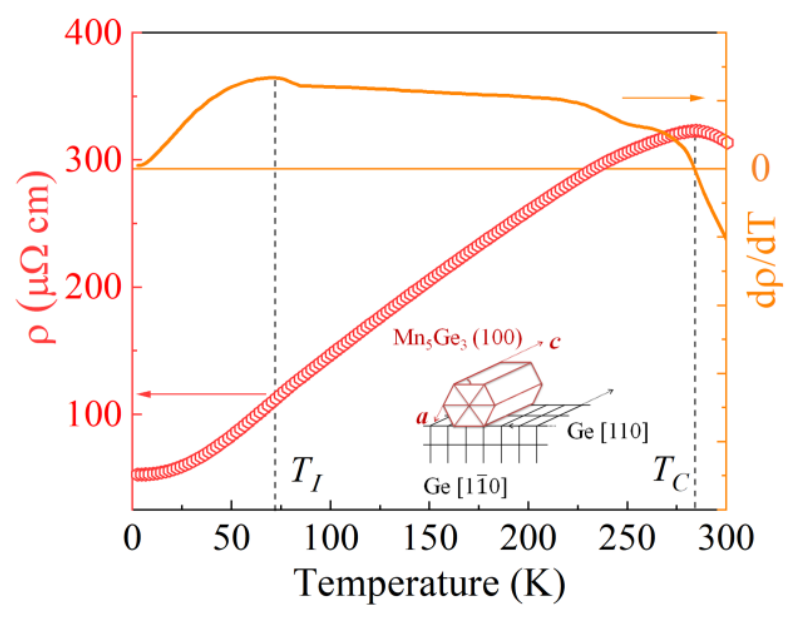

FIG. 1. Temperature dependence of the resistivity for a (100)-oriented $\mathrm{Mn}_{5} \mathrm{Ge}_{3}$ film grown on Ge (001) substrate (red circles) and the corresponding first derivative (yellow curve). The inset in Fig. 1 shows the schematic alignment of the $\mathrm{Mn}_{5} \mathrm{Ge}_{3}$ and $\mathrm{Ge}$ unit cells where the hexagonal $\mathrm{Mn}_{5} \mathrm{Ge}_{3}(100)$ is twisted with respect to the $\langle 110>$ of cubic Ge (001). Here, we only show one case where the $c$ axis of $\mathrm{Mn}_{5} \mathrm{Ge}_{3}$ is roughly oriented along the [110] direction of Ge.

The AMR is a response of the electronic structure in magnetic materials to the variation of the magnetization direction. In traditional AMR theory of ferromagnetic metals [34,35], the magnetoresistance exhibits maximum value when the current is parallel to the magnetization direction, in which carriers moving along the magnetized direction are experiencing the 
strongest scattering potential among all Fermi surface states, whereas it shows a minimum when the current flow is perpendicular to the magnetization direction. This theory is valid for polycrystalline and amorphous ferromagnets in which the AMR only depends on the relative orientation of magnetization and current. However, in the case of epitaxial or strongly textured crystalline ferromagnetic films, such as $\mathrm{Mn}_{5} \mathrm{Ge}_{3}$ grown on $\mathrm{Ge}$, the AMR depends also on the orientation of magnetization with respect to the crystalline axes. The AMR can be decomposed into two parts, noncrystalline and crystalline terms [28,36-39]. For the noncrystalline component the AMR depends on the angle between the current and magnetization, but is independent of the orientation of magnetization with respect to the crystalline axes. On the other hand, the crystalline component relies on the symmetry of the crystal structure and is independent of the current direction.
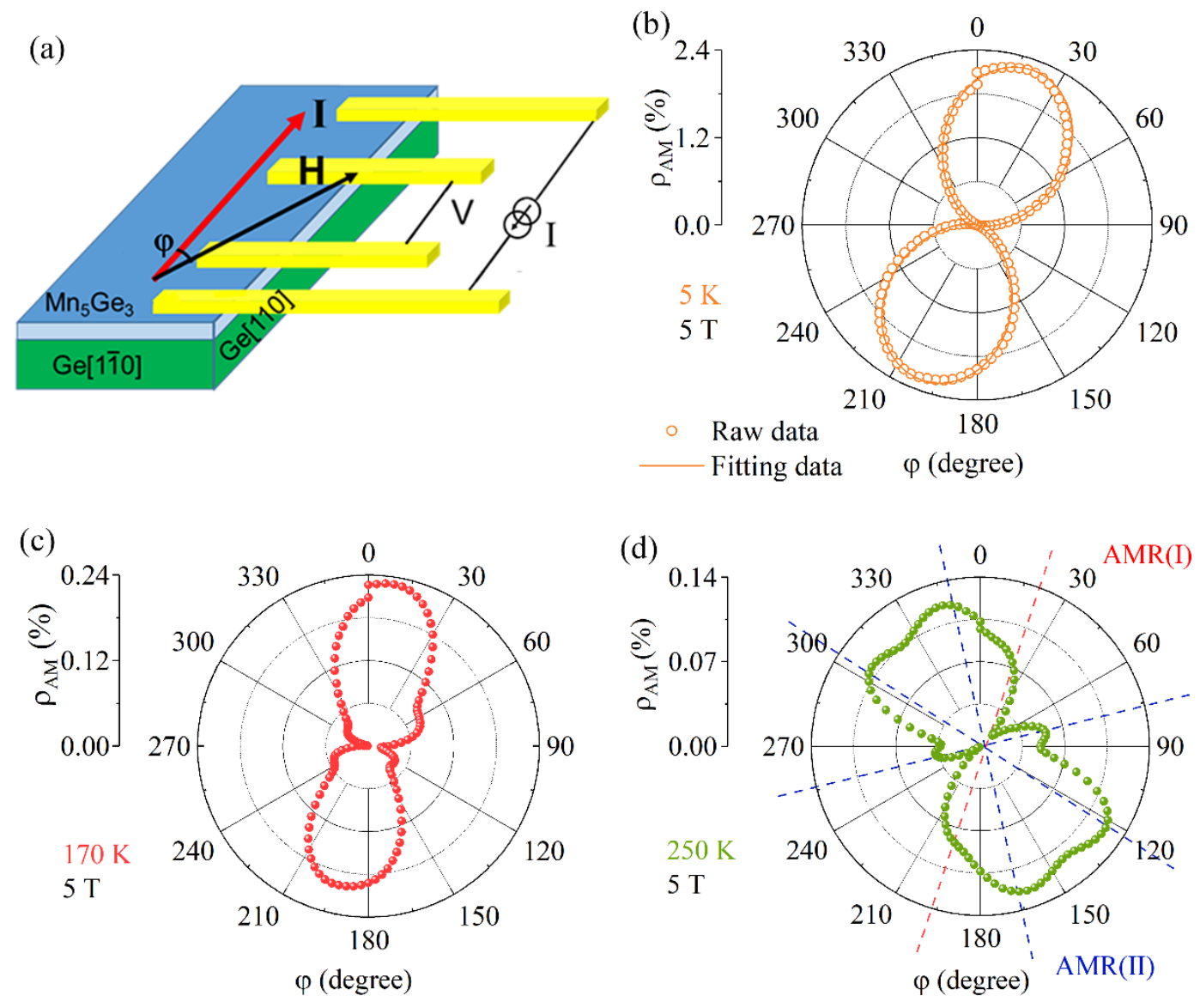

FIG. 2. (a) Schematic representation of the angular dependent magnetoresistance measurements geometry. The electric current I flows parallel to the [110] direction of Ge and the magnetic field was rotated in the surface plane (001) of Ge. The angular dependent magnetoresistance $\rho_{A M}$ with in-plane magnetic field of $5 \mathrm{~T}$ as a function of the angle $\left(\varphi\right.$ ) between magnetic field and current were measured at (b) $5 \mathrm{~K}$, (c) $170 \mathrm{~K}$, (d) $250 \mathrm{~K}$. $\rho_{A M}$ is defined as $\left[\left(\rho(\varphi)-\rho_{\min }\right) / \rho_{\min }\right] \times 100 \%$. 
Magneto-transport measurements were performed using four-wire contacts designed in a row [see Fig. 2(a)]. Figures 2(b)-2(d) show the angle $(\varphi)$-dependent anisotropic magnetoresistance $\rho_{A M}$ measured at various temperatures with magnetic field applied in-plane sample surface. As shown in Fig. S2, the AMR maxima and minima of (100)-oriented $\mathrm{Mn}_{5} \mathrm{Ge}_{3}$ are almost independent of the current flow direction but strongly dependent on the crystalline axis, which indicates dominating crystalline AMR. Therefore, the observed evolution of anisotropy in magnetoresistance can be tentatively attributed to the crystalline component of the AMR. The magnitude of the $\rho_{A M}$ and its dependence on $\varphi$ are quite diverse with temperature rising from 5 to $300 \mathrm{~K}$. At $5 \mathrm{~K}$ [see Fig. 2(b)], $\rho_{A M}$ has a large modulation $(2.24 \%$ ) as a function of $\varphi$ that accurately follows a typical $\cos ^{2} \varphi$ dependence. It exhibits twofold symmetry [AMR(I)] with global minimum values at about $110^{\circ}$ and $290^{\circ}$ while global maximum values are observed at about $20^{\circ}$ and $200^{\circ}$. The current is injected along the [110] lattice plane of Ge while the AMR response at $5 \mathrm{~K}$ is originated from the ferromagnetic $\mathrm{Mn}_{5} \mathrm{Ge}_{3}$ layer. As shown in the inset of Fig. 1, the out-of-plane $[210]_{\mathrm{H}}$ direction of $\mathrm{Mn}_{5} \mathrm{Ge}_{3}$ is parallel to the $[001]_{\mathrm{C}}$ direction of $\mathrm{Ge}$ substrate (the subscript indexes $\mathrm{H}$ and $\mathrm{C}$ denote the hexagonal and cubic unit cells, respectively) [3]. The $[001]_{\mathrm{H}}$ direction of $\mathrm{Mn}_{5} \mathrm{Ge}_{3}$ lies in the surface plane of the $\mathrm{Ge}(001)$ substrate, and is roughly oriented along the $\langle 110\rangle_{\mathrm{C}}$ direction of Ge. Due to the relatively large lattice mismatch between $\mathrm{Mn}_{5} \mathrm{Ge}_{3}$ and $\mathrm{Ge}$ the unit cell of the ferromagnetic film is misoriented in all directions, resulting in the tilt of the (100) net planes and a twist in the basal plane. The tilt/rotation of the $\mathrm{Mn}_{5} \mathrm{Ge}_{3}$ layer with respect to the Ge substrate is likely related to the shift of the twofold symmetry global maximum in the AMR signal with respect to the injected current, consistent with our finding of dominating crystalline AMR. The AMR symmetry changes with increasing temperature. Most probably, this is mainly due to the change of the magnetic coupling between $\mathrm{Mn}_{2}$ atoms with increasing interatomic distance between them. The twofold AMR contributed from $\mathrm{Mn}_{1}$ atoms becomes weaker while the additional AMR component with new symmetries contributed from $\mathrm{Mn}_{2}$ becomes stronger with increasing temperature. With increasing temperature, the $\mathrm{Mn}_{2}$ atoms in the $6 g$ site become FM coupled, which changes the distribution of the local magnetic moments and results in a multifold symmetry in the AMR signal [schematically marked with blue dashed lines in Fig. 2(d)]. At this temperature, the multifold symmetry co-exists with the $\mathrm{Mn}_{1}$ related twofold symmetry is schematically shown in Fig. 2(d).

Now we discuss the AMR evolution in detail. Firstly, with increasing temperature from 5 to $150 \mathrm{~K}$, the magnitude of $\rho_{A M}$ reduces from 2.24 to $0.38 \%$, but still shows the identical twofold AMR [Fig. S3(a-c)] [28]. At 170 K [see Fig. 2(c)], besides the AMR(I) component with twofold 
symmetry, additional local peaks emerge along several specific angular directions (i.e., $\varphi=70^{\circ}$, $115^{\circ}, 160^{\circ}, 250^{\circ}, 295^{\circ}$, and $340^{\circ}$ ). At $250 \mathrm{~K}$ (see Fig. 2(d)), the magnitude of inherent twofold $\mathrm{AMR}(\mathrm{I})$ is strongly suppressed (reduced to $0.07 \%$ ), while the newly emerged AMR(II) component becomes stronger and new global maxima appear at $\varphi=75^{\circ}, 125^{\circ}, 175^{\circ}, 255^{\circ}, 305^{\circ}$, and $355^{\circ}$. At $250 \mathrm{~K}$ the AMR(II) increases up to $0.12 \%$ for the global maximum at $125^{\circ}$. With increasing temperature from 170 to $250 \mathrm{~K}$ the angle interval between maxima in AMR(II) changes from $45^{\circ}$ to $50^{\circ}$. The position of the global maximum changes with increasing temperature. Most probably, the rotation of the global maximum is due to the structural deformation of the $\mathrm{Mn}_{5} \mathrm{Ge}_{3}$ unit cell with temperature causing a change in the spatial position of the $\mathrm{Mn}$ atoms. At $300 \mathrm{~K}$, the $\mathrm{Mn}_{5} \mathrm{Ge}_{3}$ is paramagnetic and the related $\rho_{A M}$ curve exhibits a twofold symmetry again (see Fig. S4) [28].

\section{B. Structural analysis}

To explain the transition of spin configurations at $72 \mathrm{~K}$ and the diverse AMR symmetries observed at different temperature regimes, the magneto-transport properties of $\mathrm{Mn}_{5} \mathrm{Ge}_{3}$ are further discussed in terms of structural evolutions and corresponding DFT calculations.
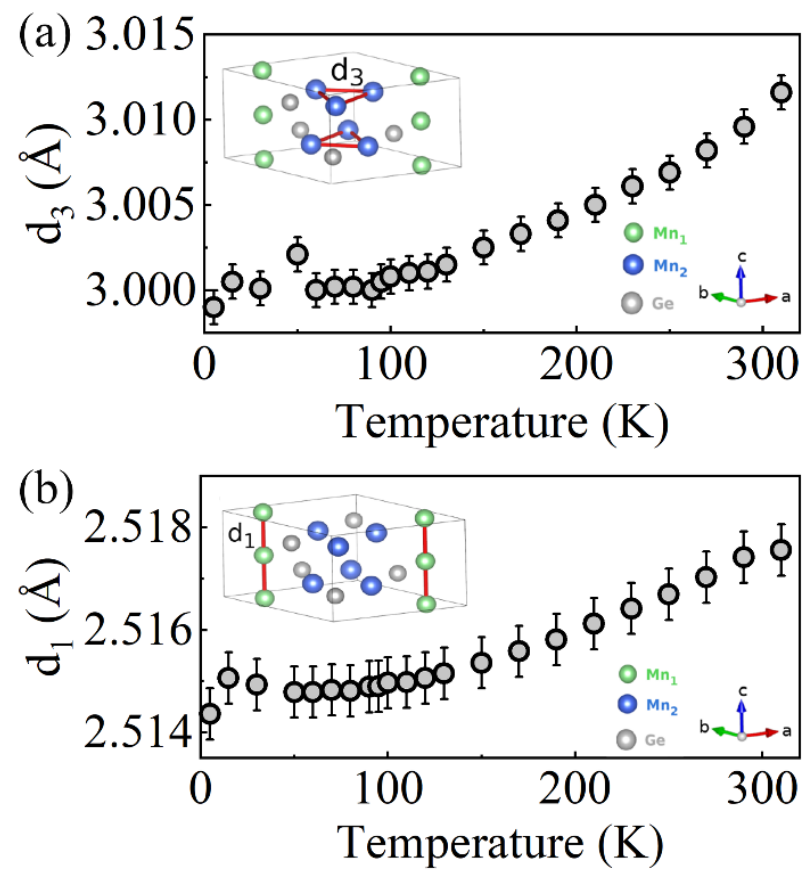

FIG. 3. (a) and (b) show temperature dependent distances $d_{3}$ and $d_{1}$ between the nearest neighbour atoms in $\mathrm{Mn}_{2}$ and $\mathrm{Mn}_{1}$ sublattices as deduced from TDXRD and tabulated lattice spacing at room temperature $\left(d_{3}^{0}=3.017 \AA, d_{1}^{0}\right.$ $=2.518 \AA$ ) [14], respectively. Error bars are shown for the measured atomic distances $d_{3}$ and $d_{1}$ while the error in the temperature is within the size of the used data symbols. The insets in (a) and (b) show the schematic picture of the crystal structure. The $\mathrm{Mn}_{1}, \mathrm{Mn}_{2}$ and Ge atoms are shown with green, blue and grey circles, respectively. 
Figures 3(a) and (b) show the distances $d_{3}$ and $d_{1}$ between the nearest neighboring atoms in $\mathrm{Mn}_{2}$ and $\mathrm{Mn}_{1}$ sublattices extracted from the TDXRD results (see Fig. S5) [28,40,41], respectively. Upon cooling, both $d_{3}$ and $d_{1}$ distinctly shrink with decreasing the temperature. At $150 \mathrm{~K}, d_{3}$ reduces to $3.002 \AA$. Here, for the calculation of $d_{3}$ we assumed an identical change of the $d_{3}$ for all $\mathrm{Mn}_{2}$ atoms in the basal configuration and a constant parameter of the $6 g$ Wyckoff position. In fact, while the basal configuration of the $\mathrm{Mn}_{2}$ unit in relaxed $\mathrm{Mn}_{5} \mathrm{Ge}_{3}$ is an equilateral triangle, only one side is aligned along the out-of-plane direction in our film geometry. Nevertheless, the assumptions made here are sufficient to present qualitatively the influence of the temperature-dependent strain and the unit-cell deformation on the AMR and spin configuration. It is important to note that the decreasing of $d_{3}$ is much faster than $d_{1}$. This is due to the different thermal expansion coefficient $(\alpha)$ for the semimetallic $\mathrm{Mn}_{5} \mathrm{Ge}_{3}$ film (the average $\left.\alpha=2.6 \times 10^{-5} \mathrm{~K}^{-1}\right)$ and for semiconducting $\mathrm{Ge}\left(\alpha=6.1 \times 10^{-6} \mathrm{~K}^{-1}\right)$ [8]. For the $\mathrm{Mn}_{5} \mathrm{Ge}_{3}$ film, the change of the out-of-plane lattice parameter is about four times larger than the change of the in-plane lattice parameter that depends on the Ge substrate. As a result, the hexagonal unit cell is flattened and distorted at lower temperatures, where the $\mathrm{Mn}_{5} \mathrm{Ge}_{3}$ unit cell has the large $c / a$ ratio.

\section{Density-functional theory (DFT) calculations}
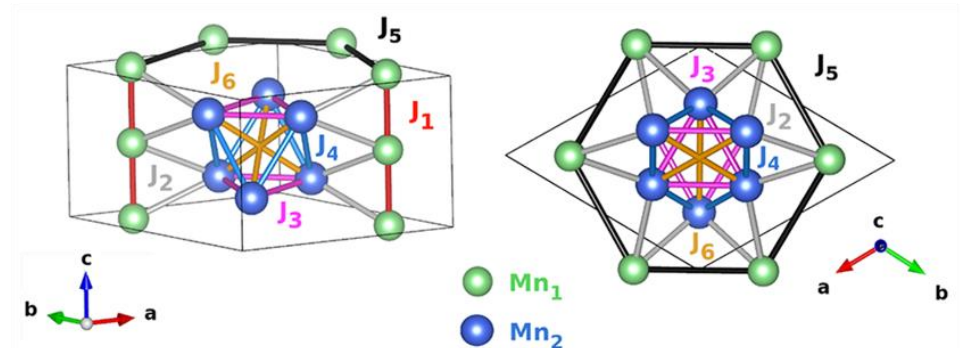

FIG. 4. Schematic picture of the crystal structure and exchange couplings. Different colours indicate various MnMn distances and the corresponding exchange couplings. The thin black lines denote the size of the unit cell. The $\mathrm{Mn}_{1}$ and $\mathrm{Mn}_{2}$ atoms in the sublattices with fourfold and sixfold positions are marked with green and blue colour. The Ge atoms are not shown here and the unit cell is shifted to $(0.5,0.5,0)$.

To understand the roles of accumulated strains on the magneto-transport properties of experimental samples, we carried out the computations [42-46] assuming the relative change of the lattice values obtained from the experiments. In order to gain insight into macroscopic magnetism revealed in the experimental samples, it is crucial to examine the microscopic picture. Note that the macroscopic magnetism originates from short-range exchange couplings that constitute the microscopic magnetic model. Moreover, any microscopic magnetic model is 
apparently related to the geometrical features of the underlying crystal structure, in particular, the mutual arrangements of the magnetic atoms. Thus, it is crucial to consider the distances between the nearest-neighboring Mn ions in each of two sublattices for the magnetic ground state $\left(\mathrm{M}_{1}\right.$ configuration in Fig. S6) [28]. $\mathrm{Mn}_{5} \mathrm{Ge}_{3}$ films invariably present ferromagnetic behavior with the exertion of experimental strain, in accordance with previously reported results $[15,16]$.

Furthermore, we examine the impact of the elastic strains on the exchange interactions between the Mn spins (shown in Fig. 4), by considering an effective classical spin Hamiltonian [46]. The details of these calculations along with derived equations are presented in Supplemental Material [28] (see Figs. S6-8).

Table I. Exchange couplings for the strained $\mathrm{Mn}_{5} \mathrm{Ge}_{3}$ samples at $5 \mathrm{~K}$ and unstrained $\mathrm{Mn}_{5} \mathrm{Ge}_{3}$. Negative and positive $J_{\mathrm{ij}}$ values denote AFM and FM couplings, respectively. The changes in distances are given in respect to the FM ground state.

\begin{tabular}{ccc}
\hline$J_{\mathrm{ij}}\left(d_{\mathrm{ij}}\right)(\mathrm{meV})$ & $\begin{array}{c}\text { Strained } \\
\text { at } 5 \mathrm{~K}\end{array}$ & Unstrained \\
\hline$J_{1}^{\mathrm{Mn} 1-\mathrm{Mn} 1}\left(\Delta d_{1}\right)$ & $33.2(0.003 \AA)$ & 33.6 \\
$J_{2}^{\mathrm{Mn} 1-\mathrm{Mn} 2}$ & 6.9 & 8.4 \\
$J_{3}^{\mathrm{Mn} 2-\mathrm{Mn} 2}\left(\Delta \mathrm{d}_{3}\right)$ & $-0.2(0.017 \AA)$ & 1.0 \\
$J_{4}^{\mathrm{Mn} 2-\mathrm{Mn} 2}$ & 5.7 & 5.7 \\
$J_{5}^{\mathrm{Mn} 1-\mathrm{Mn} 1}$ & 8.2 & 8.5 \\
$J_{6}^{\mathrm{Mn} 2-\mathrm{Mn} 2}$ & 4.8 & 4.8 \\
\hline
\end{tabular}

Note that the nearest-neighbor interaction between the $\mathrm{Mn}_{1}-\mathrm{Mn}_{1}$ exhibits FM exchange coupling $J_{1}$, which dominates over the remaining interactions. See Table I. The negligible changes in $J_{1}$ are reflected by the very small changes of $d_{1}$ distances, which are approximately $0.003 \AA$ between 300 and $5 \mathrm{~K}$. In the case of the $\mathrm{Mn}_{2}$ sublattice the AFM coupling appears under the compressive strain. The AFM interaction is more favorable for the $\mathrm{Mn}_{2}$ sublattice when the $\mathrm{d}_{3}$ distance is getting shortened, in agreement with previously reported calculations [16]. However, note that in Ref. [16] the authors reported a larger change of $J_{3}$ [from -2.04 (rigid) to $1.80 \mathrm{meV}$ (relaxed)] which corresponds to a larger shortening of the $\mathrm{d}_{3}$ distance $(0.083$ $\AA$ presented in Ref. 16 vs $0.017 \AA$ A obtained in this work). The latter is probably a consequence 
of different and larger strain used in comparison to our simulations, not directly stated in [16]. According to our DFT calculation, for $d_{3}$ larger than $3.002 \AA$ the $\mathrm{Mn}_{2}$ sublattice exhibits ferromagnetism. However, the $\mathrm{Mn}$ atoms in the $\mathrm{Mn}_{2}$ sublattice are AFM coupled for the $d_{3}$ smaller than $3.002 \AA$.

\section{Discussion}

Finally, we propose a scenario for the noncollinear to collinear switching and anomalous AMR behavior as a function of temperature. During the cooling process, $d_{3}$ becomes smaller than $3.002 \AA$ at a temperature below $150 \mathrm{~K}$ (see Fig. 3). Furthermore, the FM coexists with AFM states at low temperatures in $\mathrm{Mn}_{5} \mathrm{Ge}_{3}$ films. Since the AFM coupling in the $\mathrm{Mn}_{2}$ sublattice induces the change of local spin ordering, it would exert different influence on the AMR. In addition, it has been reported that the electronic properties of $\mathrm{Mn}_{5} \mathrm{Ge}_{3}$ depend strongly on the atomic distance and atomic environment of Mn atoms [28]. In this case, the magnetoresistance below $150 \mathrm{~K}$ with a twofold symmetry can be related to the interaction between conduction electrons and $\mathrm{FM}$ spin ordering in the $\mathrm{Mn}_{1}$ sublattice. In the temperature range from 150 to 280 $\mathrm{K}$ the AMR shows combination of both twofold and multifold symmetry. We infer that the twofold symmetry is caused by FM coupling between Mn atoms with fourfold position in the $\mathrm{Mn}_{1}$ sublattice. The multi-fold symmetry is probably associated with the FM coupling between $\mathrm{Mn}_{2}$ atoms with sixfold position in the $\mathrm{Mn}_{2}$ sublattice.

In addition, the coexistence of AFM and FM coupling observed at low-temperatures probably contributes to a low temperatures noncollinear spin configuration which is commonly observed in $\mathrm{Mn}_{5} \mathrm{Ge}_{3}$ thin films. With temperature decreasing below $150 \mathrm{~K}$, the AFM coupling between $\mathrm{Mn}_{2}$ atoms is increasing. So, the magnetic state depends on the distance between the Mn atoms, and spins will rotate to adjust to the new positions of the atoms with temperature decreasing. Upon cooling to $70 \mathrm{~K}$, the strong enough AFM coupling can compete with FM coupling, and thus it is highly plausible to result in the noncollinear spin state. Moreover, Stroppa and Peressi have shown that the noncollinear spin configuration in $\mathrm{Mn}_{5} \mathrm{Ge}_{3}$ is stabilized at a higher $c / a$ ratio [15]. In our work, $\mathrm{Mn}_{5} \mathrm{Ge}_{3}$ exhibits a larger $c / a$ ratio with decreasing temperature, which additionally justifies the existence of noncollinear spin configuration below $70 \mathrm{~K}$. The experimental error in the presented atomic distances might also contribute to the discrepancy between these two identified characteristic temperatures.

\section{CONCLUSION}


In summary, the ferromagnetic $\mathrm{Mn}_{5} \mathrm{Ge}_{3}$ films grown on $\mathrm{Ge}$ substrates show anomalous temperature-dependent AMR and switching from noncollinear to collinear spin configuration. Using different experimental techniques combined with DFT calculations, we have proposed a physical scenario responsible for both anomalous AMR and the switch of the spin configuration. The low-temperature noncollinear spin configuration very probably arises from the coexistence of AFM and FM coupling between $\mathrm{Mn}$ atoms in the $\mathrm{Mn}_{2}$ and $\mathrm{Mn}_{1}$ sublattices. The change of the magnetic coupling between $\mathrm{Mn}$ atoms in the $\mathrm{Mn}_{2}$ sublattice from AFM to FM is proposed as an explanation for the anomalous AMR behavior as a function of temperature. Our results would stimulate further investigations to understand the intriguing magnetic properties in $\mathrm{Mn}_{5} \mathrm{Ge}_{3}$ films.

\section{Acknowledgments}

The author Y.X. (File No. 201706340054) acknowledges the financial support by China Scholarship Council. M.B. is funded by the National Science Centre Grant No. UMO2016/23/D/ST3/03446. Access to computing facilities of PL-Grid Polish Infrastructure for Supporting Computational Science in the European Research Space and of the Interdisciplinary Center of Modeling (ICM), University of Warsaw is gratefully acknowledged. M.B. made use of computing facilities of TU Dresden ZIH within the project "TransPheMat". The low temperature X-ray diffraction was performed in MGML (http://mgml.eu/), which was supported within the program of Czech Research Infrastructures (Project No. LM2018096).

\section{Author contribution}

Author Y.X. performed the basic magnetic and transport measurements. Y.Y. and C.Z. performed angular dependent magnetoresistance measurements. P.D. and D.K. performed the temperature dependent XRD measurements. M.B. performed the DFT calculations and related analyses. J.G. contributed to the discussion about structural analyses. D.K. and X.Z. contributed to the discussion about transport analyses. M.H., S.Z., L.C., Y.-J.Z. and M.W. contributed to the revision of the manuscript. S.P. designed and supervised the whole project.

\section{References}

[1] I. Žutić, J. Fabian, and S. D. Sarma, Rev. Mod. Phys. 76, 323 (2004).

[2] X. Jiang, R. Wang, R. M. Shelby, R. M. Macfarlane, S. R. Bank, J. S. Harris, and S. S. Parkin, Phys. Rev. Lett. 94, 056601 (2005).

[3] Y. Xie, Y. Yuan, M. Wang, C. Xu, R. Hübner, J. Grenzer, Y. J. Zeng, M. Helm, S. Zhou, 
and S. Prucnal, Appl. Phys. Lett. 113, 222401 (2018).

[4] Y. S. Dedkov, M. Holder, G. Mayer, M. Fonin, and A. B. Preobrajenski, J. Appl. Phys. 105, 073909 (2009).

[5] W. Ndiaye, J. M. Mariot, P. De Padova, M. C. Richter, W. Wang, O. Heckmann, A. TalebIbrahimi, P. Le Fevre, F. Bertran, C. Cacho, and M. Leandersson, Phys. Rev. B 91, 125118 (2015)

[6] A. Spiesser, F. Virot, L. A. Michez, R. Hayn, S. Bertaina, L. Favre, M. Petit, and V. Le Thanh, Phys. Rev. B 86, 035211 (2012).

[7] V. Le Thanh, A. Spiesser, M. T. Dau, S. F. Olive-Mendez, L. A. Michez, and M. Petit, Adv. Nat. Sci. Nanosci. 4, 043002 (2013).

[8] D. D. Dung, D. Odkhuu, L. Thanh Vinh, S. Cheol Hong, and S. Cho, J. Appl. Phys. 114, 073906 (2013).

[9] T. Nie, J. Tang, X. Kou, Y. Gen, S. Lee, X. Zhu, Q. He, L. T. Chang, K. Murata, Y. Fan, and K. L. Wang, Nat. Commun. 7, 12866 (2016)

[10] M. Gajdzik, C. Sürgers, M. Kelemen, H. V. Löhneysen, and M. Materials, J. Magn. Magn. Mater. 221, 248 (2000).

[11] A. Spiesser, I. Slipukhina, M. T. Dau, E. Arras, V. L. Thanh, L. A. Michez, P. Pochet, H. Saito, S. Yuasa, M. Jamet, and J. Derrien, Phys. Rev. B 84, 165203 (2011).

[12] R. F. Jackson, R. G. Scurlock, D. B. Utton and E. M. Wray, Proc. Phys. Soc. 85, 127 (1965)

[13] G. Kappel, G. Fischer, and A. Jaegle, Phys. Status Solidi A 34, 691 (1976).

[14] J. Forsyth and P. J. Brown, J. Condens. Matter Phys. 2, 2713 (1990)

[15] A. Stroppa and M. Peressi, Phys. Status Solidi A, 20444 (2007).

[16] I. Slipukhina, E. Arras, P. Mavropoulos, and P. Pochet, Appl. Phys. Lett. 94, 192505 (2009).

[17] J. M. D. Coey, Can. J. Phys. 65, 1210 (1987).

[18] Y. O. Kvashnin, W. Sun, I. Di Marco, and O. Eriksson, Phys. Rev. B 92, 134422 (2015).

[19] C. Zeng, S. C. Erwin, L. C. Feldman, A. P. Li, R. Jin, Y. Song, J. R. Thompson, and H. H. Weitering, Appl. Phys. Lett. 83, 5002 (2003). C. Zeng, Y. Yao, Q. Niu, and H. H. Weitering, Phys. Rev. Lett. 96, 037204 (2006)

[20] J. Tang, C.Y. Wang, W. Jiang, L.T. Chang, Y. Fan, M. Chan, C. Wu, M. H. Hung, P. H. Liu, H. J. Yang, and H. Y. Tuan, Nano Lett. 12, 6372 (2012).

[21] P. J. Brown, J. B. Forsyth, V. Nunez, and F. Tasset, J. Condens. Matter Phys. 4, 10025 (1992). P. J. Brown and J. B. Forsyth, J. Condens. Matter Phys. 7, 7619 (1995).

[22] J. Leciejewicz, B. Penc, A. Szytuła, A. Jezierski, and A. Zygmunt, Acta Phys. Pol. A 113, 
1193 (2008).

[23] C. Surgers, G. Fischer, P. Winkel, and H. V. Löhneysen, Nat. Commun. 5, 3400 (2014).

[24] P. Hohenberg and W. Kohn, Phys. Rev. 136, B864 (1964).

[25] W. Kohn and L. J. Sham, Phys. Rev. 140, A1133 (1965).

[26] J. P. Perdew, K. Burke, and M. Ernzerhof, Phys. Rev. Lett. 77, 3865 (1996).

[27] S. Bechler, M. Kern, H. S. Funk, G. Colston, I. A. Fischer, D. Weißhaupt, M. Myronov, J. Van Slageren, and J. Schulze, Semicond. Sci. Technol. 33, 095008 (2018).

[28] See Supplemental Material at [URL will be inserted by publisher] for the discussion of temperature dependent magnetization, the AMR measurement details and more AMR results and discussion, TDXRD measurement details and analysis of the related raw data, theoretical calculations and additional results.

[29] C. Sürgers, G. Fischer, P. Winkel, and H. V. Löhneysen, Phys. Rev. B 90, 104421 (2014).

[30] S. Picozzi, A. Continenza, and A. J. Freeman, Phys. Rev. B 70, 235205 (2004).

[31] N. Yamada, J. Phys. Soc. 59, 273 (1990).

[32] A. Z. Menshikov, A. P. Vokhmyanin, and Y. A. Dorofeev, Phys. Status Solidi B 158, 319 (1990).

[33] H. Reichlová et al. https://arxiv.org/abs/2012.15651.

[34] J. Smit, Physica 17, 612 (1951).

[35] T. McGuire and R. L. Potter, IEEE T. Magn. 11, 1018 (1975).

[36] W. Döring, Ann. Phys. 424, 259 (1938)

[37] B. Howells, M. Wang, K. Edmonds, P. Wadley, R. Campion, A. Rushforth, C. Foxon, and B. L. Gallagher, Appl. Phys. Lett. 102, 052407 (2013).

[38] R. P. Van Gorkom, J. Caro, T. M. Klapwijk, and S. Radelaar, Phys. Rev. B 63, 134432 (2001).

[39] Y. Yuan, R. Hübner, M. Birowska, C. Xu, M. Wang, S. Prucnal, R. Jakiela, K. Potzger, R. Böttger, S. Facsko, J. A. Majewski, M. Helm, M. Sawicki, S. Zhou, and T. Dietl, Phys. Rev. Mater. 2, 114601 (2018).

[40] M. S. Henriques, D. I. Gorbunov, D. Kriegner, M.Vališka, A.V.Andreev, and Z. Matěj, J. Magn. Magn. Mater. 400, 125 (2016).

[41] D. Kriegner, Z. Matěj, R. Kužel, and V. Holý, J. Appl. Crystallogr. 48, 613 (2015).

[42] G. Kresse and J. Hafner, Phys. Rev. B 47, 558 (1993).

[43] G. Kresse and J. F. Mueller, Comput. Mater. Sci. 6, 15 (1996). 
[44] P. E. Blochl, Phys. Rev. B 50, 17953 (1994).

[45] G. Kresse and D. Joubert, Phys. Rev. B 59, 1758 (1999).

[46] S. L. Dudarev, G. A. Botton, S. Y. Savrasov, C. J. Humphreys, and A. P. Sutton, Phys. Rev. B 57, 1505 (1998). 